\title{
Designing of Lab View Based Electrical Capacitance Tomography System for the Imaging of Bone Using NI ELVIS and NI USB DAQ 6009
}

\author{
Bify Baby Abraham and G. Anitha
}

\begin{abstract}
Electrical Capacitance Tomography (ECT) is a method for the determination of the dielectric permittivity in the interior of the object based on exterior capacitance measurements. The principle is based on inter-electrode capacitance measurements which are done by placing electrodes around the dielectric medium which is generally made of PVC and the medium that is to be imaged is placed inside this PVC cylinder. As we know capacitance varies with respect to dielectric permittivity, area of the plates and distance between the plates. In this technique area and distance between the electrodes are kept constant, so the only parameter that influences the capacitance detected is the dielectric permittivity of the medium inside there by making this technique a suitable method for distinguishing substances of differing dielectric properties. . This technique has got several advantages such as low cost, rapid response, high robustness and no radiation hazard. Till now it is used in Oil Industries for detecting bubbles in the oil flow based on the difference in their dielectric permittivity. The aim of this paper is to apply this technique in medical application by modeling an ECT system that can be used for imaging of the bone, as human tissues shows a change in the dielectric properties, which will thereby open a new path in the field of medical imaging. The entire technique is implemented with the help of LabVIEW. Results obtained shows that this technique can be used for the imaging of bone.
\end{abstract}

Keywords--- Dielectric Permittivity, ECT, Single Electrode Excitation, Labview

\section{INTRODUCTION}

$\mathrm{T}$ HE term tomography as we know refers to the process of exploring the internal characteristics of a specified region through integral measurements related to the internal characteristics of the specified domain. Usually this term is refers to those processes that obtain $2 \mathrm{D}$ cross sections called tomograms. Recent researches in this field resulted in the development of 3D and 4D imaging techniques which provide more detailed and accurate data's. The application of LabVIEW in ECT has bring out rapid developments in this field ([1],[7]). Generally tomography is classified into 2 types. They are Direct Tomography and Indirect Tomography. In the

Bify Baby Abraham, Student, Biomedical, SRM University, Chennai, India.E-mail: bifybabraham@gmail.com

G. Anitha, Assistant Professor, Biomedical, SRM University, Chennai, India. E-mail: mailtoanithagovindhan@gmail.com direct method, visual recording not visible to human eyes are used. For example X-ray imaging, where as in indirect method boundary measurements related to the internal characteristics of the object of interest is made for image reconstruction. However an acceptable tomographic technique for medical application is the one which should posses the following properties (1) Non-invasive: means no direct contact with the domain of interest, (2) Non-intrusive: it does not change or disturb the nature of the object being explored and (3) Radiation free. Generally for detecting any factures in bones we usually go for X-ray and CT scanning's. As we know medical imaging techniques such as CT, X-ray imaging makes use of radiations which has got immense harmful effects. Radiation exposure is a concern in both adults and children. However, these concerns are greater for children because they are more sensitive to radiation and have a longer life expectancy than adults. It's found out that accumulated exposures over a child's lifetime are more likely to result in an adverse health effect. A child's smaller size also has an impact on the radiation dose they receive. For example, if a CT scan is performed on a child using the same parameters as those used on an adult, an unnecessarily large dose will be delivered to the child. So it's time for us to think about a more reliable and radiation free technique that can overcome the disadvantages possessed by the existing radiation techniques.ECT is such a technique that satisfies the aforementioned properties for a suitable medical imaging system and it posses the most important advantage when compared to CT and X-rays that its radiation free. Till now this technique is limited to Oil field industries for the monitoring of bubbles in oil flow based on the difference in their dielectric properties[3].Through this paper we aim to prove that ECT technique can be used for imaging of bone as we know that human tissues also differ in their dielectric properties. We implemented the idea with the help of LabVIEW and its acquisition hardware's.

\section{PRINCIPLE}

The principle of ECT is the measurement of the capacitances between the electrodes located on the exterior of the region of interest. Since area and distance between the plates are constant in this technique the capacitance detected will depend on the permittivity value of the material located between the electrodes. Substances of differing dielectric properties can be distinguished by means of this method. To obtain a spatially resolved image of the permittivity distribution inside the pipe, multiple electrodes are arranged on the tube and all inter-electrode capacitances are measured 
as shown in Fig1. As we know capacitance mainly depends on area of the plate, distance between plates and dielectric constant. Here dielectric constant only changes which result in capacitance change. The main principle governing the system is given below.

$$
C=A \varepsilon / \mathrm{d}
$$

where $\mathrm{C}$ is the capacitance measured $\mathrm{A}$ is the area of the plates, $\mathrm{d}$ is the distance between plates and $\varepsilon$ is the dielectric permittivity of the medium between the plates.

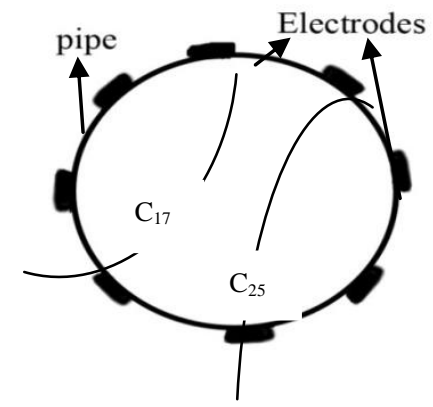

Figure 1: Electrode Arrangement and Inter Electrode Capacitance

Generally in ECT we use 8, 16 or 32 electrodes. In this paper we designed an 8 electrode acquisition system.

\section{ECT MODEL EQUATION}

The three main equations governing the designing of electrodes for an ECT system are given below [2].

$$
\begin{aligned}
& \nabla . \varepsilon \nabla \varphi(\mathrm{x}, \mathrm{y})=0 \\
& \mathrm{E}=-\nabla \varphi(\mathrm{x}, \mathrm{y}) \\
& \mathrm{C}_{\mathrm{ij}}=\mathrm{Q}_{\mathrm{ij}} / \Delta \mathrm{V}
\end{aligned}
$$

Where $\nabla \varphi(\mathrm{x}, \mathrm{y})$ is the scalar potential function, $\mathrm{Q}_{\mathrm{ij}}$ is interpreted as the charge induced on conductor $\mathrm{i}$ due to the potential difference $\mathrm{V}_{\mathrm{i}}-\mathrm{V}_{\mathrm{j}}$.

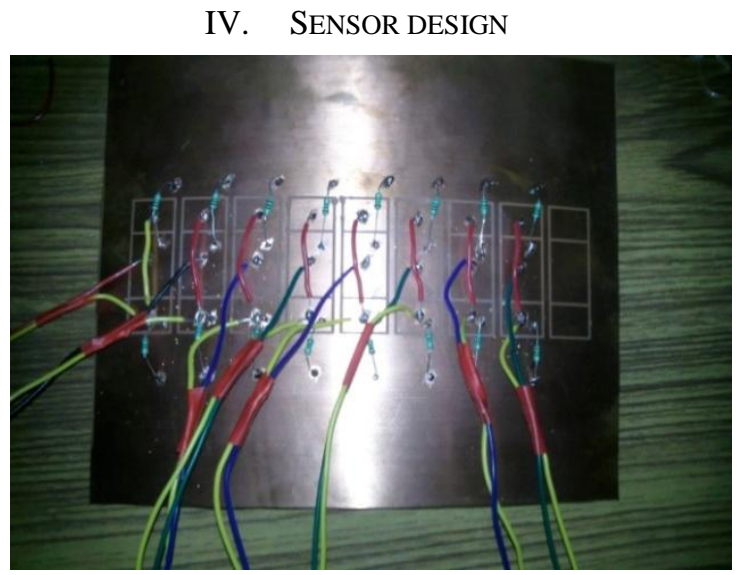

Figure 2: Electrodes Etched on the FR4 Glass Fiber Coated with Copper
The sensing unit designed is an 8 electrode system. These 8 electrodes are etched on an FR4 glass fibre coated with copper as shown in Fig2. The design procedures are explained below.

Capacitance measurements are made between each electrode and every other electrode. For a sensor containing $\mathrm{E}$ measurement electrodes, there are $\mathrm{E}$ (E-1)/2 unique capacitance measurements. This corresponds to 28 individual measurements for our 8 electrode sensor. Because the capacitance values are very small, the various design parameters of the ECT sensor head are important and can be summarized as follows [2].

1. Thickness and the material of the wall between the electrodes and the sensing zone (the insulating lining).

2. Thickness and the material of the wall between the electrodes and the screen.

3. Size of the electrodes.

4. Guarding used.

5. Gap between the electrodes and the guards, and the dimensions of the guarding electrodes.

While designing the electrodes the insulation lining between the electrodes is of greater importance. If the insulation lining between the electrodes is too large then the inter-electrode capacitance detected will be very low even though the permittivity over the cross section is large. Driven guard electrodes are placed in front and back of the original electrodes as shown in Fig3, which are driven by the same potential as the main electrode. Between these electrodes and the original ones, there are small gaps that insulate the circuits from each other. These electrodes help in providing a uniform electric field distribution by the main sensor.

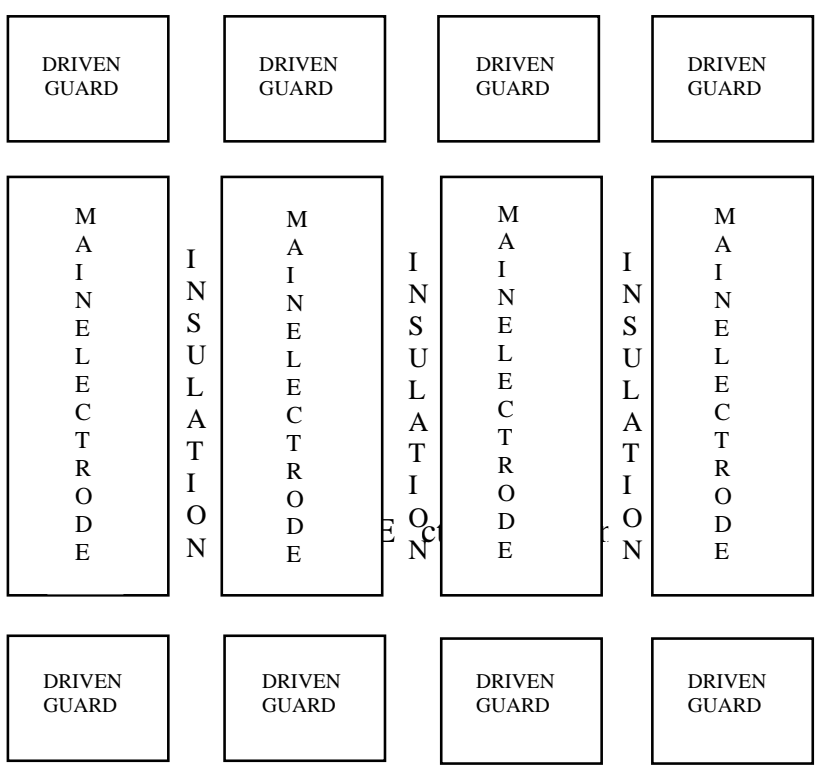

Before the designing of electrodes we should keep one factor in our mind that in order to get best axial resolution we should use small number of measurement electrodes. But in order for getting a superior cross sectional images we should go for large number of measurement electrodes. The orthogonal resolution will be approximately $\mathrm{D} / \mathrm{E}$ where $\mathrm{D}$ is 
the sensor diameter and $\mathrm{E}$ is the number of measuring electrodes. If the tube wall thickness is greater than around 5\% of the tube diameter, there is a danger that the adjacent capacitance values will decrease as the permittivity of the material inside the sensor increases ([8],[6]). This is more likely to occur for sensors with larger number of electrodes.

The total length of the electrode including driven guard and the measurement electrode should at least be equal to the diameter of the pipe on which it is mounted for support. Once the total length of the measurement electrode is fixed, the length of the driven guard electrode can be found out using the formula [8].

$$
\mathrm{Lt}=\mathrm{Lm}+2 . \mathrm{Lg}
$$

Where Lt is the total electrode length, $\mathrm{Lm}$ is the length of the measurement electrodes and $\mathrm{Lg}$ is the length of the driven guard electrodes. Usually the minimum length chosen for the measurement electrode for an 8electrode system mounted on a 2 inch pipe is $35 \mathrm{~mm}$.As per Fig2 we can see 9 electrodes etched on the FR4 glass fibre coated with copper. Here the 9th electrode is acting as a dummy electrode which is used to provide symmetry. No connections will be given to this extra electrode. Each measurement and guard electrode must be connected to an earthed area on the sensor laminate by an individual discharge resistor. Generally we use $1 \mathrm{Mohm}$ resistors.

1. Pipe outer diameter $=2.4$ inches $=6.2 \mathrm{~cm}=62 \mathrm{~mm}$

2. Pipe inner diameter $=2.3$ inches $=5.9 \mathrm{~cm}=59 \mathrm{~mm}$

3. Total length of the electrode $[\mathrm{Lt}]=$ measurement electrode length $[\mathrm{Lm}]+2$ (guard electrode length) $[\mathrm{Lg}]$

4. $\mathrm{Lg}=12 \mathrm{~mm}$

5. Width of the single electrode $=15.643 \mathrm{~mm}$

6. Width of the insulating layer $=1 \mathrm{~mm}$

7. Axial guard width $=4 \mathrm{~mm}$

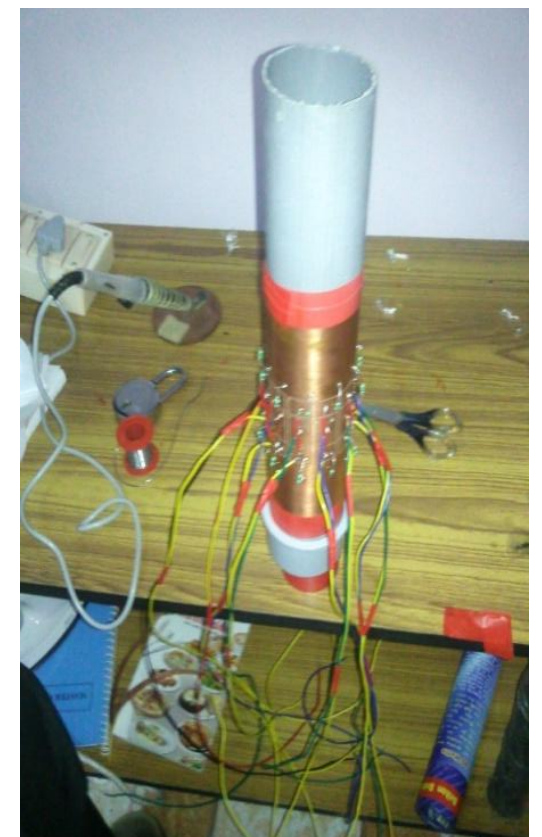

Figure 4: PVC Pipe Wrapped with Electrodes
After soldering the necessary connections and discharge resistors on the driven guard and main electrodes, the FR4 glass fibre is then wrapped around the PVC pipe as shown in Fig. 4 which will act as the sensing unit for the application.

\section{MethodOLOGY}

The technique used to excite the 8 electrode system is the single electrode excitation method [5]. But the method used here is slightly different from the conventional single electrode excitation method. In this method we will excite one electrode at a time and the inter electrode capacitance existing between this electrode with respect to the other electrodes is measured with the help of LabVIEW data acquisition hardware NI USB DAQ 6009.After this, in the next step the second electrode will be excited and the inter-electrode capacitance between this electrode with respect to other electrodes are measured. The cycle continues till the last electrode in which 7th electrode will be excited and the interelectrode capacitance between 7th and 8th electrode is measured. Note that the inter-electrode capacitance once measured won't be measured again during the same cycle. For example the inter-electrode capacitance between 1st and 2nd will be measured while exciting the first electrode and we won't measure the inter electrode capacitance between 2nd and 1st while we are exciting the 2nd electrode. At the end of one cycle we will have a set of 28 measurements and the cycle continues. The excitation pattern is shown in Table 1.

Table 1: Excitation Pattern

\begin{tabular}{|c|l|}
\hline Electrode Excited & \multicolumn{1}{|c|}{ Capacitance measured } \\
\hline 1 & $\mathrm{C}_{12}, \mathrm{C}_{13}, \mathrm{C}_{14}, \mathrm{C}_{15}, \mathrm{C}_{16}, \mathrm{C}_{17}, \mathrm{C}_{18}$ \\
\hline 2 & $\mathrm{C}_{23}, \mathrm{C}_{24}, \mathrm{C}_{25}, \mathrm{C}_{26}, \mathrm{C}_{27}, \mathrm{C}_{28}$ \\
\hline 3 & $\mathrm{C}_{34}, \mathrm{C}_{35}, \mathrm{C}_{36}, \mathrm{C}_{37}, \mathrm{C}_{38}$ \\
\hline 4 & $\mathrm{C}_{45}, \mathrm{C}_{46}, \mathrm{C}_{47}, \mathrm{C}_{48}$ \\
\hline 5 & $\mathrm{C}_{56}, \mathrm{C}_{57}, \mathrm{C}_{58}$ \\
\hline 6 & $\mathrm{C}_{67}, \mathrm{C}_{68}$ \\
\hline 7 & $\mathrm{C}_{78}$ \\
\hline
\end{tabular}

Before the implementation of excitation pattern the sensitivity of the system should be checked first. Along with that we have to find out the necessary amplitude that should be possessed by the excitation signal in order to excite the electrodes. So as the first step, 1st electrode is excited with a $5 \mathrm{~V}$ pulse signal from USB DAQ and the inter-electrode capacitance of the 1st electrode with respect to the others is measured. Similarly other electrodes are excited separately with the same pulse and checked for the inter-electrode capacitance, but it was found that noise is dominating the detected signal. Example of a waveform obtained when exciting the electrode with a $5 \mathrm{~V}$ pulse is shown in Fig5. As we know a capacitor will charge to the voltage we are supplying across it. The maximum output voltage that can be given using NI USB DAQ 6009 is 5V. So for checking with higher voltages function generator of NI ELVIS II is used. The amplitude of the signal is varied and the inter-electrode capacitance is detected through the NI ELVIS II for various amplitude levels. It was found that for voltage levels above 9, we are getting detectable output voltages. These detected voltages are proportional to the capacitance variation inside 
the electrode system. We know that the maximum input voltage for USB DAQ 6009 is $10 \mathrm{~V}$, and it also lacks an isolation amplifier. Since its maximum input range is $10 \mathrm{~V}$, the excitation pulse signals amplitude is fixed to 9V.The systems sensitivity to frequency variation is then checked. The $9 \mathrm{~V}$ signal required for the excitation is produced with the help of a rectifier circuit as shown in Fig 6.

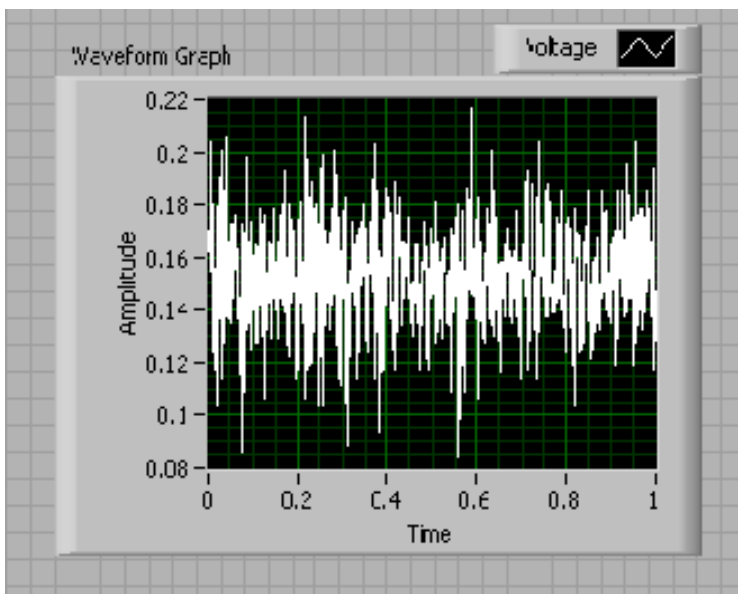

Figure 5: Data Detected when 1st Electrode is Excited with a Pulse Signal of Amplitude 5V and Detected Through 2nd Electrode

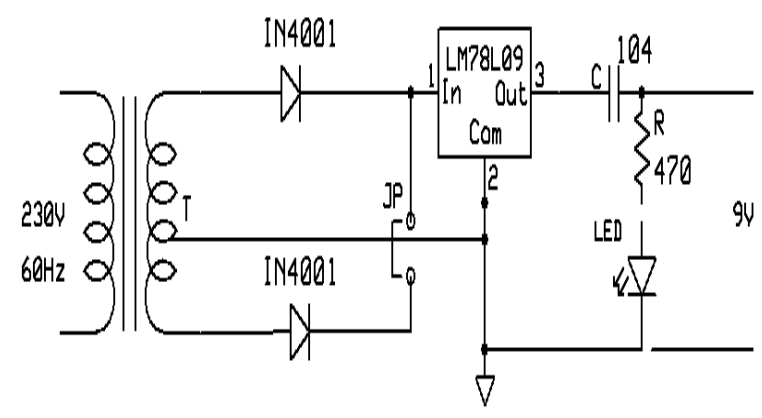

Figure 6: Rectifier Circuit

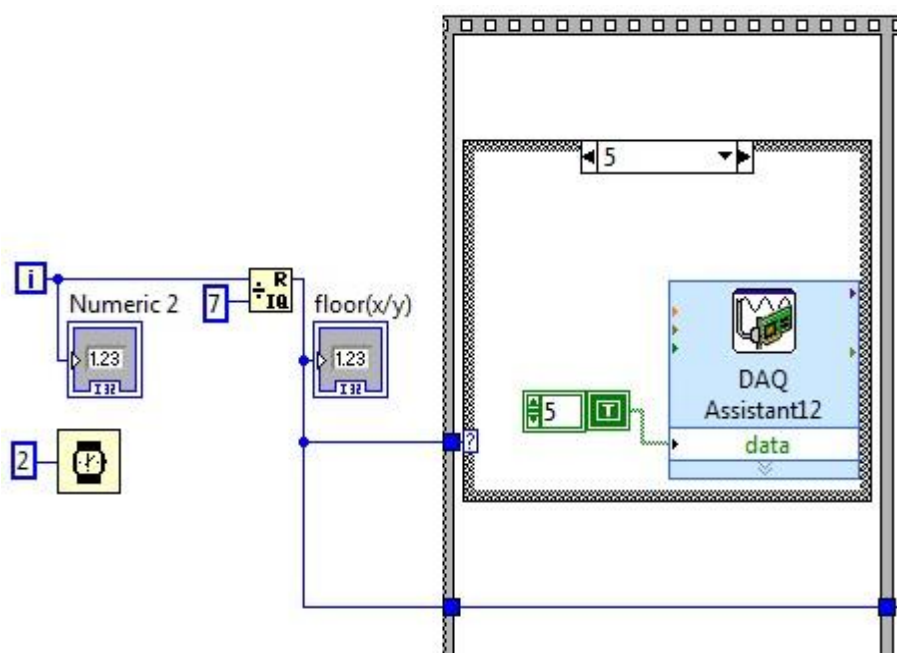

Figure 7: Electrode Excitation Logic Developed in Lab view
Electrode excitation and data acquisition logic is developed with the help of LabVIEW 2010.The excitation and detection are done with the help of case structures. Excitation and acquisition logic developed in LabVIEW are shown in Fig 7 and Fig 8 respectively.

\section{RESUlTS AND DiSCUSSIONS}

Signal detected via NIELVIS II (when an excitation voltage of $10 \mathrm{~V}$ is given form the NI ELVIS II Function generator) when air is inside the electrode system is shown in Fig 9.The important thing to be remembered is the inverse relationship between voltage and capacitance. So a high permittivity medium will have high capacitance value and hence less voltage. Similarly a low permittivity medium will have a less capacitance value, but the voltage detected will be high. The voltage detected for water is less compared to voltage detected for air and bone. The voltage detected for bone is much higher than air as its having low permittivity when compared with air. The signal detected for bone is shown in Fig. 10

The next step is to develop image from the detected values. Sensitivity matrix for different electrodes is developed and the detected values are mapped to it. The resultant images for water inside a bottle and the bovine bone are shown in Fig11 and Fig 12 respectively. High permittivity medium will be having low voltage detected and hence it has been assigned red and for low permittivity medium voltage detected is high and it has been given green colour.

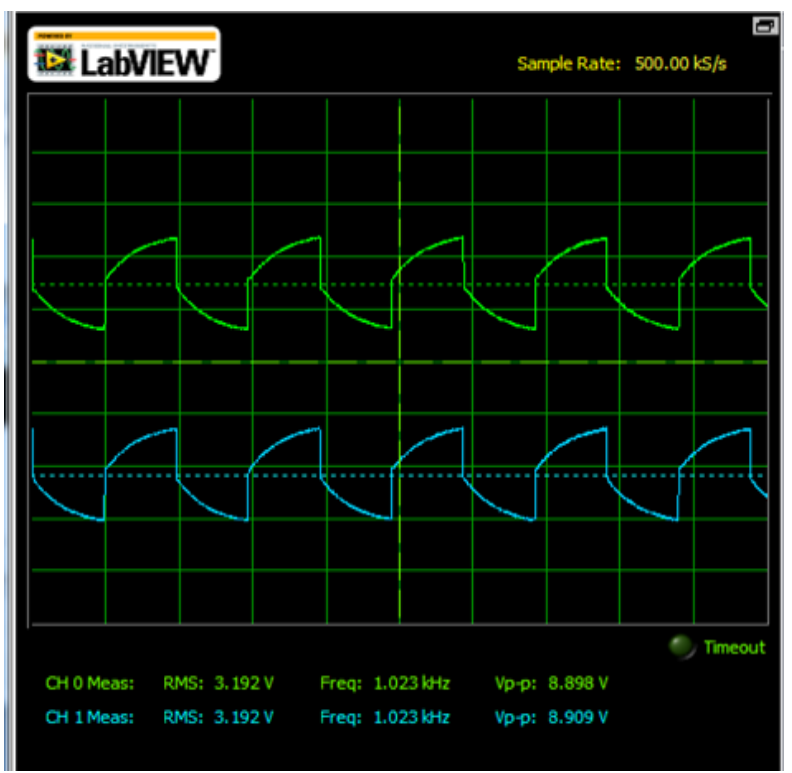

Figure 9: Data Detected when 1st Electrode is Excited with a 9V Signal and Detected through 2nd and 3rd Electrodes when Air is Inside the System 


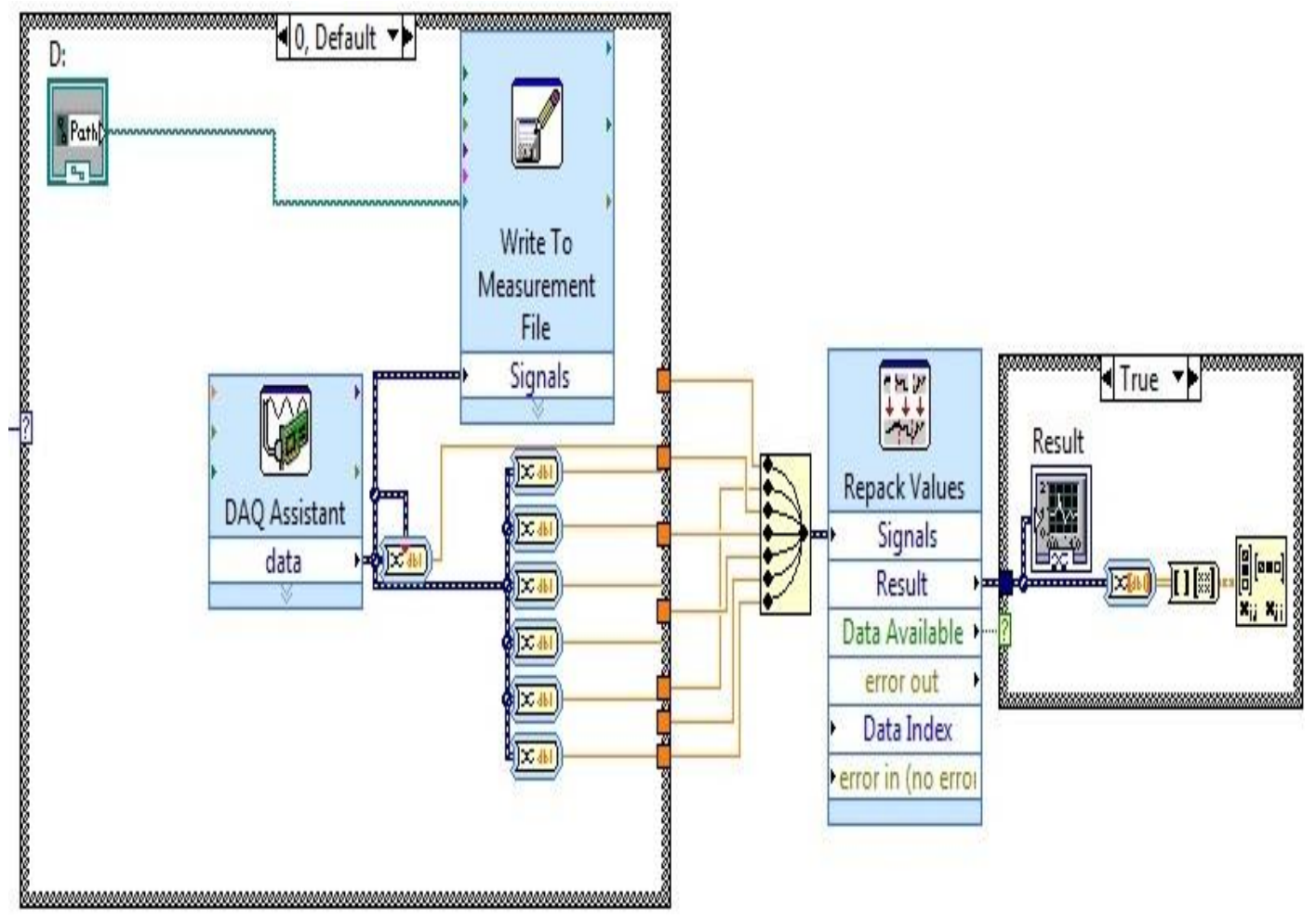

Figure 8: Acquisition Logic Developed using Lab view

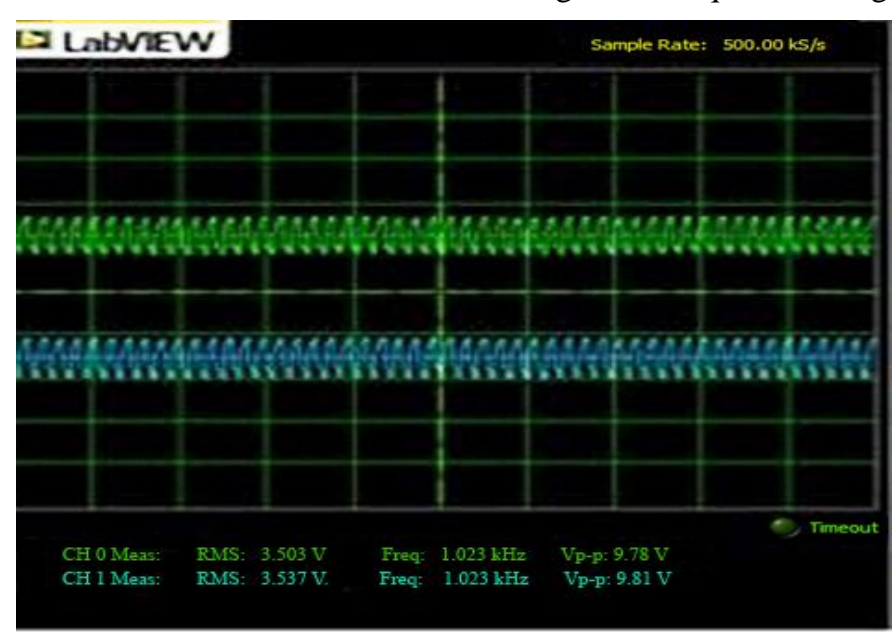

Figure 10: Data Detected when $1^{\text {st }}$ Electrode is Excited with a 9V Signal and Detected Through $2^{\text {nd }}$ and $3^{\text {rd }}$ Electrode when Bone is Placed Inside the System

\section{CONCLUSION AND FUTURE ENHANCEMENT}

The results obtained, point towards the fact that ECT technique can be used for the imaging of human tissues also. But in order to get much higher resolution we should design high speed acquisition devices that can detect data's in nanoseconds and also the electrode number should be increased to 16 or more. If we incorporate ECTs 4D modeling technique [4], which has been already developed for Oil industries to this, then we can look forward for a new and advanced imaging modality which can revolutionize the field of medical imaging.

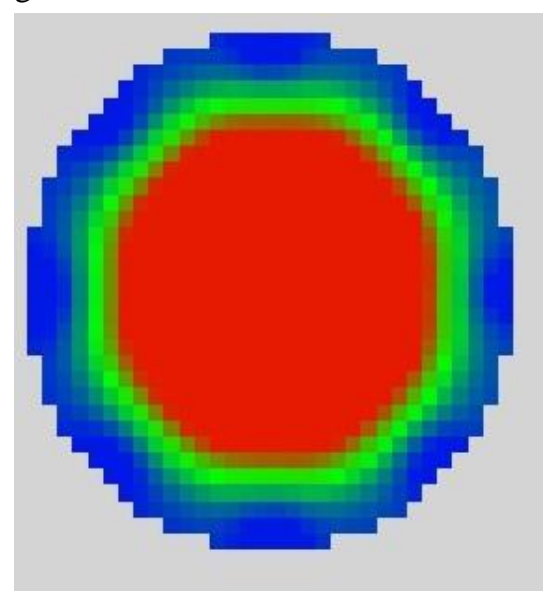

Figure 11:Image of Water in a Bottle

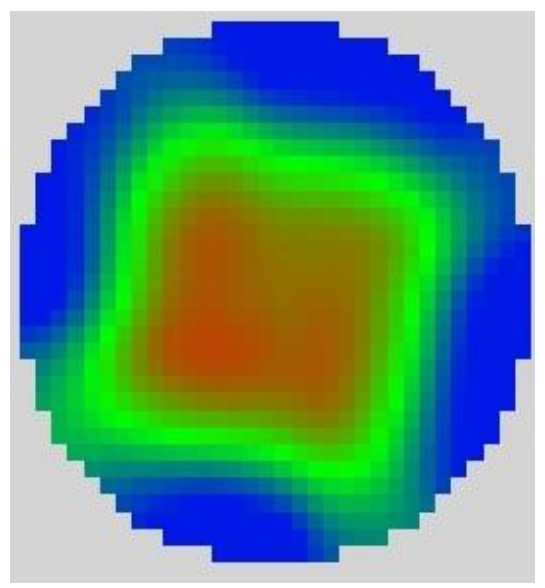

Figure 12: Image of Bone when Placed Inside the Electrode System 


\section{ACKNOWLEDGMENT}

We sincerely thank Mr. Joyce Palliyath and Mr.Sivanathan for their guidance. We also thank the faculties of Biomedical Department, SRM University for their constant support and prayers.

\section{REFERENCES}

[1] Zhaoyan Fan, Robert X. Gao* and Jinjiang Wang "Virtual instrument for online electrical capacitance tomography", Textbook on LabVIEW practical applications and solutions, 2011.

[2] Kjell Joar Alme and Saba Mylvaganam, "Electrical Capacitance Tomography - Sensor Models, Design, Simulations, and Experimental verification", IEEE Sensor Journal, Vol 6, October 2006.

[3] Kenneth R. Foster, "Dielectric Properties of Tissues", The Biomedical Engineering Handbook, $2^{\text {nd }}$ Edition, Chapter 89,2000.

[4] Warsito Warsito, Qussai Marashdeh, Liang-Shih Fan "Electrical Capacitance Volume Tomography”, IEEE sensors journal, Vol. 7, No. 4, April 2007.

[5] S.Sathyamoorthy and J.Sarachandrababu, "Design of High-Speed Pulse Input Based Capacitance Measurement for Electrical Capacitance Tomography", Sensors \& Transducers Journal, Vol.75, Issue 1, January 2007.

[6] Yandi Gao, Qing Xu, Feng Zhou,Jiang Xi and NanChang "Research on Factors of Influencing ECT Image Reconstruction", Second IEEE Conference on Industrial Electronics and Applications,2007.

[7] W. Q. Yang and J. Kjærsgaard Rasmussen "A Compact Electrical Capacitance Tomography System", IEEE International Workshop on Imaging Systems and Techniques - IST, September 10-12, 2008

[8] (2001) [Online]. Available: http:// www.tomography.com.

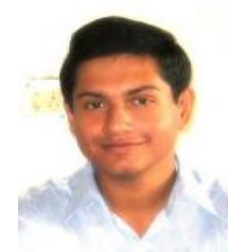

Bify Baby Abraham received his B.Tech Degree in Biomedical Engineering from Sahrdaya College of Engineering and Technology, Thrissur, Kerala, India in the year 2010. He completed his Masters in Biomedical Engineering from SRM University, Chennai in the year 2012. His field of interest is in Medical Imaging. He is born on $7^{\text {th }}$ December 1988. He had successfully completed trainings in LabVIEW from NI Bangalore.

He had published paper named 'Electrical Capacitance Tomography' in SJCET journal of Engineering and Management in the year 2010. 